\title{
Pliocene-Pleistocene radiolarian and diatom biostratigraphy of ODP Hole 709C (equatorial Indian Ocean)
}

\author{
Simon K. Haslett ${ }^{\prime}$, Kevin Kennington ${ }^{2}$, Brian M. Funnell ${ }^{2}$ \& Claire L. Kersley ${ }^{3}$ \\ ${ }^{\prime}$ Faculty of Applied Sciences, Bath College of Higher Education, Newton Park, Newton St. Loe, Bath BA2 9BN, UK \\ ${ }^{2}$ School of Environmental Sciences, University of East Anglia, Norwich NR4 7TJ, UK \\ ${ }^{3}$ Grafham Carbons, Grafham Water Works, Perry, Huntingdon PE18 OBW, UK
}

\begin{abstract}
Ocean Drilling Program (ODP) Hole 709C was drilled on Madingley Rise in the equatorial Indian Ocean. Thirteen radiolarian and diatom events were identified in the Pliocene to Early Pleistocene section studied. Five radiolarian zones could be recognized: Anthocyrtidium angulare, Pterocanium prismatium, Anthocyrtidium jenghisi, Stichocorys peregrina, and Phormostichoartus doliolum zones. The LAD of $P$. prismatium was found to be an unreliable indicator of the base of the $A$. angulare zone in Hole $709 \mathrm{C}$ and in other upwelling areas. Accordingly, the base of the $A$. angulare zone is redefined here by the FAD of $A$. angulare. The Phormostichoartus fistula zone, which normally separates the $S$. peregrina and $P$. doliolum zones, could not be identified due to the scarcity of $P$. fistula. Standard tropical diatom Pliocene-Pleistocene zones and subzones were identified: Nitzschia reinholdii, Rhizosolenia praebergonii, and Nitzschia jouseae zones. The development of oxygen isotope calibrated dates enabled the dating of some radiolarian events: FAD A. angulare $1.65 \mathrm{Ma}$, LAD Theocorythium vetulum 2Ma, LAD A. jenghisi $2.27 \mathrm{Ma}$, FAD Theocalyptra davisiana $2.33 \mathrm{Ma}$, FAD Theocorythium trachelium $2.33 \mathrm{Ma}$, and FAD Lamprocyrtis neoheteroporos 2.4 Ma. J. Micropalaeontol. 14(2): 135-143, October 1995.
\end{abstract}

\section{INTRODUCTION}

The primary objective behind the drilling of Site 709 in the equatorial Indian Ocean, was to retrieve a continuous Neogene sediment sequence from which carbonate preservation variability throughout time could be assessed. Hole $709 \mathrm{C}$ was one of three holes drilled at the Site, with $327.9 \mathrm{~m}$ of core recovered at a total recovery rate of $93 \%$, spanning the Eocene through to Recent. The first 20 cores representing the topmost $189.1 \mathrm{~m}$ of sediment, achieved almost $100 \%$ core recovery (Shipboard Scientific Party, 1988).

Most shipboard and post-cruise micropalaeontological work was carried out on calcareous microfossils: calcareous nannofossils (Fornaciari et al., 1990; Matsuoka \& Okada, 1990; Okada, 1990; Rio et al., 1990), and foraminifera (Boersma, 1990; Premoli-Silva \& Spezzaferri, 1990; Haslett \& Kersley, 1995). Siliceous microfossils were also investigated: radiolaria (Johnson, 1990; Haslett, 1992; Haslett et al., 1994) and diatoms (Mikkelsen, 1990; Fenner \& Mikkelsen, 1990; Kennington, 1992). From the three holes drilled at Site 709 , Johnson (1990) concentrated on 709A to provide the Pliocene-Pleistocene radiolarian biostratigraphy, whilst using $709 \mathrm{C}$ to provide biostratigraphical data for EoceneOligocene sediments. Mikkelsen (1990) investigated the Pliocene-Pleistocene diatom biostratigraphy of 709C, but the resolution was low with some samples spaced more than $4 \mathrm{~m}$ apart. Fenner \& Mikkelsen (1990) studied only Eocene-Oligocene diatoms.

The main purpose of the present study was to provide detailed biostratigraphical information, using radiolarian and diatom datum levels. The $709 \mathrm{C}$ radiolarian data are then compared with a recent palaeomagnetically constrained radiolarian biostratigraphical scheme from the Indian Ocean (Johnson et al., 1989).

At the present time the surface oceanography of the equatorial Indian Ocean is influenced by the seasonal variation of the monsoonal gyre. The seasonal changes involve the complete reversal of the prevailing wind field between the northeast and southwest monsoons. A distinct hydrochemical front, located approximately $10^{\circ} \mathrm{S}$ (Wyrtki, 1973), separates upwelling waters of the reversing gyre to the north from surface waters of the subtropical gyre to the south. North of the hydrochemical front (Site 709 is situated near $4^{\circ} \mathrm{S}$ ), the surface waters possess higher nutrient levels and support correspondingly higher productivity. In the subtropical gyre both nutrient levels and productivity are low. The higher concentration of biogenic opal present in sediment north of $10^{\circ} \mathrm{S}$ (Leinen et al., 1986) is linked with the higher nutrient and productivity levels of the surface waters. This scenario is probably valid at least as far back as the middle Miocene (Johnson, 1990).

\section{MATERIAL \& METHODS}

Hole $709 \mathrm{C}$ was drilled as part of Ocean Drilling Program (ODP) Leg 115, and is located in the equatorial Indian Ocean, in a small basin near the summit of Madingley Rise, a regional topographic high between the Carlsberg Ridge and the western Mascarene Plateau. Its position is Latitude $03^{\circ} 54.9^{\prime} \mathrm{S}$, Longitude $60^{\circ} 33.1^{\prime} \mathrm{E}$.

At Hole $709 \mathrm{C}$ the water depth is $3040.8 \mathrm{~m}$ with bottom felt (distance from rig floor to sea floor) at $3048.7 \mathrm{~m}$. The total length of cored section was $353.7 \mathrm{~m}$ (to $3391.9 \mathrm{~m}$ ) with $327.9 \mathrm{~m}$ of total core recovered. The samples used for Pliocene-Pleistocene radiolarian and diatom slide prepara- 
tion range from $3 / 1 / 5-7 \mathrm{~cm}$ (hydraulic piston core/APC No. 3) to $6 / 2 / 5-7 \mathrm{~cm}$ (APC 6), spanning a depth in metres below sea-floor from $15.45 \mathrm{mbsf}$ to $45.85 \mathrm{mbsf}$.

The basic lithology consisted of foraminifera-bearing nannofossil ooze, largely homogenous but with some bioturbation. The sediment varies in colour from very light grey, through light greenish grey to white with some pyrite-stained burrows in sections 1 and 3 of Core 3 . Core 5 possesses 26 thin interbedded horizons of very light grey and light greenish grey foraminifera-bearing nannofossil ooze concentrated in sections 2 and 4 . Core 6 possesses 35 thin interbedded horizons of very light grey, light grey and very light greenish grey foraminifera-bearing nannofossil ooze distributed throughout the core (Shipboard Scientific Party, 1988).

Samples were provided as $<150 \mu \mathrm{m}$ dried residues (Andrews et al., 1990). Normally, $1 \mathrm{~g}$ of sediment was processed. Aliquots of the complete $<150 \mu \mathrm{m}$ fraction were used in the preparation of slides for diatom analysis, whilst a $63-150 \mu \mathrm{m}$ fraction was used to concentrate radiolaria (Mullineaux \& Westberg-Smith, 1986; Haslett \& Robinson, 1991). $1 \mathrm{~g}$ of sediment from each sample was placed in $10 \mathrm{ml}$ plastic test-tubes. Dilute hydrochloric acid $(\mathrm{HCl})$ was added and agitated until all the carbonate had been dissolved. When the reaction was complete (no more effervescence) the samples were washed with distilled water to dilute and remove the $\mathrm{HCl}$. The samples were then centrifuged and the supernatant decanted. This process was repeated before $10 \mathrm{ml}$ of the deflocculent sodium hexametaphosphate (in solution) was added. The sample was again centrifuged and the supernatant decanted. Further rinsing, centrifuging and decanting was carried out until the sediment was seen to be 'clean'.

A small quantity of sample was withdrawn with one squeeze of a pipette and then released onto a glass coverslip. The coverslips were left to dry on an even surface (i.e. photographic paper, which does not warp when wet). When dry, a pea-sized blob of Naphrax (Northern Biological Supplies, Ipswich) was pipetted onto a labelled glass microscope slide. The slide was inverted and used to pick up the coverslip using the natural tackiness of the mountant. Slides were placed on a warm hotplate to enable the mounting medium to spread evenly and also to expel any air bubbles (without allowing the mountant to boil). When the mounting medium reached the edges of the coverslip, the slides were removed from the hotplate and left to set. Two or three slides were prepared for each sample in case of breakage. These slides were then used for diatom analysis.

To concentrate radiolaria the remaining sediment in the plastic test-tubes were thoroughly washed through a $63 \mu \mathrm{m}$ sieve. After washing, distilled water was used to channel the remaining $63-150 \mu \mathrm{m}$ fraction into labelled storage vials or beakers. Slides were prepared as above with the exception that Canada Balsam was used as the mounting agent. In the first instance, slides were examined at regularly spaced core intervals of no more than $2 \mathrm{~m}$ apart. Additional slides were then examined to identify radiolarian and diatom datum levels more accurately.

The sample processing method employed here reflects the essentially non-quantitative approach taken in this particular biostratigraphical study and differs from processing techniques used for palaeoenvironmental analysis.

\section{BIOSTRATIGRAPHY \\ Radiolaria}

Radiolarian zonal schemes relating to the tropical Indian Ocean are few in comparison with those proposed for the tropical Pacific (Nigrini, 1971; Johnson \& Knoll, 1975; Riedel \& Sanfilippo, 1978; Theyer et al., 1978; Goll, 1980; Nigrini, 1985). Johnson (1990) employed a standard low latitude Cenozoic radiolarian zonation (Sanfilippo et al., 1985), although in many instances this proved unsatisfactory. Partly in response to problems recognized in applying the zonation of Sanfilippo et al. (1985) to equatorial Indian Ocean Pliocene-Pleistocene sediments, Johnson et al. (1989) proposed a new zonation calibrated by palaeomagnetic age data. The Quaternary part of the zonation is essentially similar to the standard zonation; however, the Late Pliocene has been divided into six zones, which are equivalent to only two zones of the standard zonation.

This biostratigraphical refinement has largely been made possible by taxonomic reviews of the radiolarian genera Anthocyrtidium (Nigrini \& Caulet, 1988) and Pterocorys (Caulet \& Nigrini, 1988). Unfortunately, due to the sporadic occurrence of a number of species belonging to these genera, some standard radiolarian events cannot be identified at Site 709 (Johnson, 1990). These events include the LAD (last appearance datum) of Anthocyrtidium nosicaae Caulet, FAD (first appearance datum) of $A$. euryclathrum Nigrini \& Caulet, FAD of Pterocorys zancleus (Mueller), LAD of $A$. ehrenbergi (Stohr), and the LAD of A. prolatum (Nigrini \& Caulet). Johnson (1990) noted that Theocorythium vetulum Nigrini was also virtually absent and that members of the Spongaster lineage, S. berminghami (Campbell \& Clark) to $S$. pentas Riedel \& Sanfilippo to $S$. tetras Ehrenberg, were very rare or absent. Pterocanium prismatium Riedel was also found to be rare.

\section{Diatoms}

Schrader (1974) and Kazarina (1975) were the first workers to propose diatom zonal schemes for the tropical Indian Ocean. However, it is now possible to employ the cosmopolitan low-latitude zonation of Burckle (1972), which was originally proposed for the eastern equatorial Pacific. Barron (1985) updated Burckle's scheme, and this version is used here. A coarse diatom biostratigraphy of $709 \mathrm{C}$ was given by Mikkelsen (1990).

\section{RESULTS}

Ten radiolarian and three diatom datum levels were identified (Table 1). Range and abundance data for radiolaria are given in Table 2 , and for diatoms in Table 3. Preservation of both radiolaria and diatoms is generally good, however certain poorly preserved horizons were encountered, e.g. 5/1/45-47-5/2/105-107 and 4/4/145$4 / 5 / 145-147$, which cause difficulty in assigning some datum levels. 


\begin{tabular}{ll}
\hline Species & \multicolumn{1}{c}{ Samples } \\
\hline LAD Anthocyrtidium michelinae & $<3 / 1 / 5-7$ \\
LAD Nitzschia reinholdii & $<3 / 1 / 5-7$ \\
LAD Pterocanium prismatium & $<3 / 1 / 5-7$ \\
LAD Rhizosolenia praebergonii & $<3 / 1 / 5-7$ \\
FAD Anthocyrtidium angulare & $3 / 1 / 5-7-3 / 1 / 65-67$ \\
LAD Theocorythium vetulum & $3 / 2 / 140-142-3 / 2 / 145-147$ \\
LAD Anthocyrtidium jenghisi & $3 / 4 / 5-7-3 / 4 / 75-77$ \\
FAD Theocalyptra davisiana & $3 / 4 / 145-147-3 / 5 / 75-77$ \\
FAD Theocorythium trachelium & $3 / 4 / 145-147-3 / 5 / 75-77$ \\
FAD Lamprocyrtis neoheteroporos & $3 / 5 / 75-77-3 / 5 / 145-147$ \\
LAD Thalassiosira convexa & $4 / 1 / 10-12-4 / 1 / 50-52$ \\
LAD Nitzschia jouseae & $4 / 1 / 100-102-4 / 1 / 120-122$ \\
LAD Stichocorys peregrina & $4 / 1 / 105-107-4 / 1 / 145-147$ \\
FAD Rhizosolenia praebergonii & $4 / 1 / 145-147-4 / 2 / 5-7$ \\
LAD Lychnodictyum audax & $5 / 2 / 105-107-5 / 2 / 145-147$ \\
LAD Phormostichoartus doliolum & $5 / 2 / 145-147-5 / 3 / 35-37$ \\
FAD Amphirhopalum ypsilon & $5 / 6 / 5-7-5 / C C / 5-7$ \\
FAD Spongaster tetras & $>6 / 2 / 5-7$ \\
\hline
\end{tabular}

$<$, above; $>$, below

Table 1. Position of radiolarian and diatom data in Hole $709 \mathrm{C}$.

\section{Early Pliocene}

Spongaster tetras was present in all samples examined; its FAD occurs below the studied section. Amphirhopalum ypsilon Haeckel is first encountered in $5 / 6 / 5-7$, and is present in all subsequent samples. Phormostichoartus doliolum (Riedel \& Sanfilippo) is common in samples up to its last appearance at 5/3/35-37. Lychnodictyum audax Riedel was present in only two samples, 5/6/5-7 and $5 / 2 / 145-147$.

\section{Late Pliocene}

Rhizosolenia praebergonii Mukhina is first encountered in $4 / 2 / 5-7$. Stichocorys peregrina (Riedel) is very common in all samples up to $4 / 2 / 145-147$, after which it becomes rare, and is last encountered in 4/1/145-147. Nitzschia jouseae Burkle and Thalassiosira convexa Mukhina, both of which occur consistently, are last encountered in 4/1/120-122 and $4 / 1 / 50-52$ respectively. The evolutionary transition of Lamprocyrtis neoheteroporos Kling (2 segmented) from its ancestor $L$. heteroporos (Hays) (3 segmented) is often difficult to determine; however, undoubted specimens of $L$. neoheteroporos are present in 3/5/75-77, after which it is a common faunal component. Theocorythium trachelium (Ehrenberg) was first encountered in 3/4/75-77; however, it occurs rarely and sporadically thereafter, and is often poorly preserved. Theocalyptra davisiana (Ehrenberg) is also first encountered in $3 / 4 / 75-77$, but does not become common until 3/3/65-67. Anthocyrtidium jenghisi Streeter is common up to $3 / 5 / 75-77$, after which it becomes rare, and last appears in $3 / 4 / 75-77$. Theocorythium vetulum is extremely rare throughout the section. Its last appearance occurs in $3 / 2 / 145-147$.

\section{Pleistocene}

Anthocyrtidium angulare Nigrini is first encountered in the youngest sample examined, $3 / 1 / 5-7$, although it is scarce in this sample (only two specimens seen). Pterocanium prismatium was only encountered in two samples, 3/3/65-67 and $3 / 2 / 140-142$. The LAD of this species normally post-dates the FAD of $A$. angulare (therefore $3 / 2 / 140-142$ is not taken to represent the LAD of $P$. prismatium). It is likely that the true LAD $P$. prismatium event occurs above $3 / 1 / 5-7$.

The LADs of Anthocyrtidium michelinae Caulet, Rhizosolenia praebergonii and Nitzschia reinholdii Kanaya \& Koizumi, which occur consistently throughout the section, also occur above $3 / 1 / 5-7$.

\section{DISCUSSION}

The radiolarian events recognized here can generally be referred to the zonal scheme proposed by Johnson et al. (1989). There are two differences however, which lead to a redefined base for the Anthocyrtidium angulare zone and to the omission of the Phormostichoartus fistula zone. Also, the relative position of the FADs of Theocorythium trachelium and Lamprocyrtis neoheteroporos in 709A (Johnson, 1990) and $709 \mathrm{C}$ (this study) are reversed with respect to Johnson et al. (1989). Johnson et al. (1989) indicate that these data are diachronous, suggesting that $T$. trachelium migrates into equatorial waters from the south, and that $L$. neoheteroporos originates in equatorial waters subsequently migrating to the south.

Anthocyrtidium angulare Total Range Zone (Nigrini, 1971; Caulet, 1979; Sanfilippo et al., 1985; Johnson et al., 1989; emend. here).

Definition. FAD to LAD of Anthocyrtidium angulare.

Remarks. This zone is usually considered an interval chronozone, the base of which is defined by the LAD of Pterocanium prismatium. However, in areas of equatorial upwelling $P$. prismatium has a sporadic occurrence and often has a last appearance which predates the FAD of $A$. angulare. This is seen at ODP Sites 677A (Alexandrovich, 1989), 709A (Johnson, 1990), 709C (this study), and 731 (Spaulding et al., 1991). It is clear therefore, that the LAD of $P$. prismatium is an unreliable indicator of the base of the $A$. angulare zone in upwelling areas, and its definition is accordingly emended here.

Pterocanium prismatium Interval Chronozone (Riedel \& Sanfilippo, 1970; emend. Riedel \& Sanfilippo, 1978; Caulet, 1979; emend. Sanfilippo et al., 1985; emend. Johnson et al., 1989; emend. here).

Definition. LAD of Anthocyrtidium jenghisi to FAD of Anthocyrtidium angulare.

709C events included in this zone: LAD of Theocorythium vetulum.

Remarks. The top of this zone is redefined here as the FAD of $A$. angulare, as the LAD of $P$. prismatium is an unreliable event in upwelling areas.

Anthocyrtidium jenghisi Interval Chronozone (non NR7 of Caulet, 1979; Johnson et al., 1989). Definition. LAD of Stichocorys peregrina to LAD of $A$. jenghisi.

709C events included in this zone: FAD of Theocalyptra 
Haslett, Kennington, Funnell \& Kersley

\begin{tabular}{|c|c|c|c|c|c|c|c|c|c|c|c|c|c|c|}
\hline SAMPLES & 1 & 2 & 3 & 4 & 5 & 6 & 7 & 8 & 9 & 10 & 11 & 12 & 13 & $\mathrm{Z}$ \\
\hline $3 / 1 / 5-7$ & C & C & + & C & $\mathrm{C}$ & $\mathrm{C}$ & - & $\mathrm{C}$ & & & & & & I \\
\hline $3 / 1 / 65-67$ & - & $\mathrm{C}$ & - & $\mathrm{C}$ & $\mathrm{C}$ & $\mathrm{C}$ & - & $\mathrm{C}$ & & & & & & II \\
\hline $3 / 1 / 120-122$ & & $\mathrm{C}$ & + & $\mathrm{C}$ & $\mathrm{C}$ & C & + & C & & & & & & \\
\hline $3 / 1 / 145-147$ & & $\mathrm{C}$ & - & $\mathrm{C}$ & $\mathrm{C}$ & C & - & $\mathrm{C}$ & & & & & & \\
\hline $3 / 2 / 135-137$ & & $\mathrm{C}$ & + & C & C & $\mathrm{C}$ & - & $\mathrm{C}$ & - & & & & & \\
\hline $3 / 2 / 140-142$ & & $\mathrm{C}$ & $\mathrm{C}$ & $\mathrm{C}$ & $\mathrm{C}$ & $\mathrm{C}$ & $\mathrm{C}$ & $\mathrm{C}$ & - & & & & & \\
\hline $3 / 2 / 145-147$ & & $\mathrm{C}$ & $\mathrm{C}$ & C & $\mathrm{C}$ & $\mathrm{C}$ & - & C & C & & & & & \\
\hline $3 / 3 / 10-12$ & & C & $\mathrm{C}$ & C & C & C & - & C & C & & & & & \\
\hline $3 / 3 / 65-67$ & & C & C & C & C & C & C & $\mathrm{C}$ & - & & & & & \\
\hline $3 / 4 / 5-7$ & & + & + & $\mathrm{C}$ & $\mathrm{C}$ & $\mathrm{C}$ & - & $\mathrm{C}$ & - & - & & & & \\
\hline $3 / 4 / 75-77$ & & + & + & $\mathrm{C}$ & $\mathrm{C}$ & $\mathrm{C}$ & - & $\mathrm{C}$ & - & + & & & & III \\
\hline $3 / 4 / 145-147$ & & + & + & $\mathrm{C}$ & $\mathrm{C}$ & $\mathrm{C}$ & - & $\mathrm{C}$ & - & + & & & & \\
\hline $3 / 5 / 75-77$ & & - & - & $\mathrm{C}$ & $\mathrm{C}$ & C & - & C & - & $\mathrm{C}$ & & & & \\
\hline $3 / 5 / 145-147$ & & & & - & C & C & - & $\mathrm{C}$ & - & $\mathrm{C}$ & & & & \\
\hline $3 / 6 / 145-147$ & & & & & C & C & - & $\mathrm{C}$ & - & $\mathrm{C}$ & & & & \\
\hline $3 / 7 / 50-52$ & & & & & $\mathrm{C}$ & $\mathrm{C}$ & - & C & - & $\mathrm{C}$ & $\overline{-}$ & & & \\
\hline $4 / 1 / 10-12$ & & & & & $\mathrm{C}$ & $\mathrm{C}$ & - & $\mathrm{C}$ & $\mathrm{C}$ & $\mathrm{C}$ & $\mathrm{R}$ & & & \\
\hline $\begin{array}{l}4 / 1 / 65-67 \\
4 / 1 / 105-107\end{array}$ & & & & & $\begin{array}{l}\mathrm{C} \\
+\end{array}$ & $\begin{array}{l}\mathrm{C} \\
\mathrm{C}\end{array}$ & $\begin{array}{l}- \\
-\end{array}$ & $\begin{array}{l}\mathrm{C} \\
\mathrm{C}\end{array}$ & $\begin{array}{l}- \\
-\end{array}$ & $\begin{array}{l}\mathrm{C} \\
\mathrm{C}\end{array}$ & - & & & \\
\hline $4 / 1 / 145-147$ & & & & & $\mathrm{C}$ & + & - & $\mathrm{C}$ & - & + & + & & & IV \\
\hline $4 / 2 / 65-67$ & & & & & $\mathrm{C}$ & + & - & $\mathrm{C}$ & - & $\mathrm{T}$ & $\begin{array}{l}+ \\
+\end{array}$ & & & iv \\
\hline $4 / 2 / 145-147$ & & & & & $\mathrm{C}$ & $\mathrm{C}$ & - & $\mathrm{C}$ & - & $\mathrm{C}$ & $\mathrm{C}$ & & & \\
\hline $4 / 3 / 145-147$ & & & & & + & C & - & + & - & + & $\mathrm{C}$ & & & \\
\hline $4 / 4 / 145-147$ & & & & & $\mathrm{C}$ & $\mathrm{C}$ & - & + & - & + & $\mathrm{C}$ & & & \\
\hline $4 / 5 / 145-147$ & & & & & + & + & - & + & - & + & + & & & \\
\hline $4 / 6 / 145-147$ & & & & & $\mathrm{C}$ & $\mathrm{C}$ & - & $\mathrm{C}$ & $\mathrm{C}$ & $\mathrm{C}$ & $\mathrm{C}$ & & & \\
\hline $4 / 7 / 60-62$ & & & & & $\mathrm{C}$ & C & - & $\mathrm{C}$ & - & $\mathrm{C}$ & $\mathrm{C}$ & & & \\
\hline $4 / \mathrm{CC} / 10-12$ & & & & & $\mathrm{C}$ & C & - & + & - & $\mathrm{C}$ & $\mathrm{C}$ & & & \\
\hline $5 / 1 / 5-7$ & & & & & + & $\mathrm{C}$ & - & + & - & + & $\mathrm{C}$ & & & \\
\hline $5 / 1 / 145-147$ & & & & & + & + & - & + & - & + & + & & & \\
\hline $5 / 2 / 65-67$ & & & & & + & + & - & $\mathrm{C}$ & - & + & t & - & & \\
\hline $5 / 2 / 105-107$ & & & & & + & + & - & + & - & + & + & - & - & \\
\hline $5 / 2 / 145-147$ & & & & & $\mathrm{C}$ & $\mathrm{C}$ & - & $\mathrm{C}$ & - & $\mathrm{C}$ & C & $\mathrm{C}$ & - & \\
\hline $5 / 3 / 35-37$ & & & & & + & $\mathrm{C}$ & - & + & - & $\mathrm{C}$ & $\mathrm{C}$ & - & $\mathrm{C}$ & $\mathrm{V}$ \\
\hline $5 / 3 / 65-67$ & & & & & + & + & - & + & - & + & + & - & $\mathrm{C}$ & \\
\hline $5 / 3 / 145-147$ & & & & & $\mathrm{C}$ & + & - & + & - & + & $\mathrm{C}$ & - & C & \\
\hline $5 / 4 / 5-7$ & & & & & + & + & - & + & - & + & $\mathrm{C}$ & - & $\mathrm{C}$ & \\
\hline $5 / 5 / 110-112$ & & & & & $\mathrm{C}$ & + & - & + & $\mathrm{C}$ & + & $\mathrm{C}$ & - & $\mathrm{C}$ & \\
\hline $5 / 6 / 5-7$ & & & & & $\mathrm{C}$ & + & - & $\mathrm{C}$ & $\mathrm{C}$ & + & $\mathrm{C}$ & $\mathrm{C}$ & $\mathrm{C}$ & \\
\hline $5 / \mathrm{CC} / 5-7$ & & & & & - & C & - & + & + & + & $\mathrm{C}$ & - & $\mathrm{C}$ & \\
\hline $6 / 1 / 10-12$ & & & & & - & $\mathrm{C}$ & - & + & - & + & $\mathrm{C}$ & - & $\mathrm{C}$ & \\
\hline $6 / 2 / 5-7$ & & & & & & + & - & + & - & + & $\mathrm{C}$ & - & $\mathrm{C}$ & \\
\hline
\end{tabular}

C, common; +, present; -, searched for but not found; R, reworked occurrence. Species 1 , Anthocyrtidium angulare; 2, Theocalyptra davisiana; 3, Theocorythium trachelium; 4, Lamprocyrtis neoheteroporos; 5 , Amphirhopalum ypsilon; 6, Spongaster tetras; 7, Pterocanium prismatium; 8, Anthocyrtidium michelinae; 9 , Theocorythium vetulum; 10, Anthocyrtidium jenghisi; 11, Stichocorys peregrina; 12, Lychnodictyum audax; 13, Phormostichoartus doliolum. Zone I, A. angulare zone; II, $P$. prismatium zone; III, $A$. jenghisi zone; IV, S. peregrina zone; V, P. doliolum zone.

Table 2. Radiolarian stratigraphy of Hole 709C.

\section{Explanation of Plate 1}

Fig. 1, 2. Stichocorys peregrina (Riedel), sample 5/3/65-67, mag. $\times 310$. Fig. 3. Theocorythium vetulum Nigrini, sample $3 / 3 / 5-7$, mag. $\times 330$. Fig. 4. Theocorythium trachelium (Ehrenberg), sample 3/3/10-12, mag. $\times 330$. Fig, 5. Anthocyrtidium jenghisi Streeter, sample 4/CC/10-12, mag. $\times 315$. Fig. 6. Anthocyrtidium michelinae Caulet, sample 4/7/60-62, mag. $\times 250$. Fig. 7. Phormostichoartus doliolum (Riedel \& Sanfilippo), sample 5/3/65-67, mag. $\times 230$. Fig. 8. Amphirhopalum ypsilon Haeckel, sample 5/3/65-67, mag. $\times 380$. Fig. 9. Theocalyptra davisiana (Ehrenberg), sample $3 / 1 / 5-7$, mag. $\times 170$. Fig. 10. Spongaster tetras Ehrenberg, sample $5 / 2 / 145-147$, mag. $\times 320$. Fig. 11. Lamprocyrtis neoheteroporos Kling, sample $3 / 3 / 5-7$, mag. $\times 320$. Fig. 12. Hemidiscus cuneiformis Wallich, sample $3 / 2 / 140-142$, mag. $\times 1000$. Fig. 13. Dipploneis sp., sample 5/2/145-147, mag. $\times 1200$. 

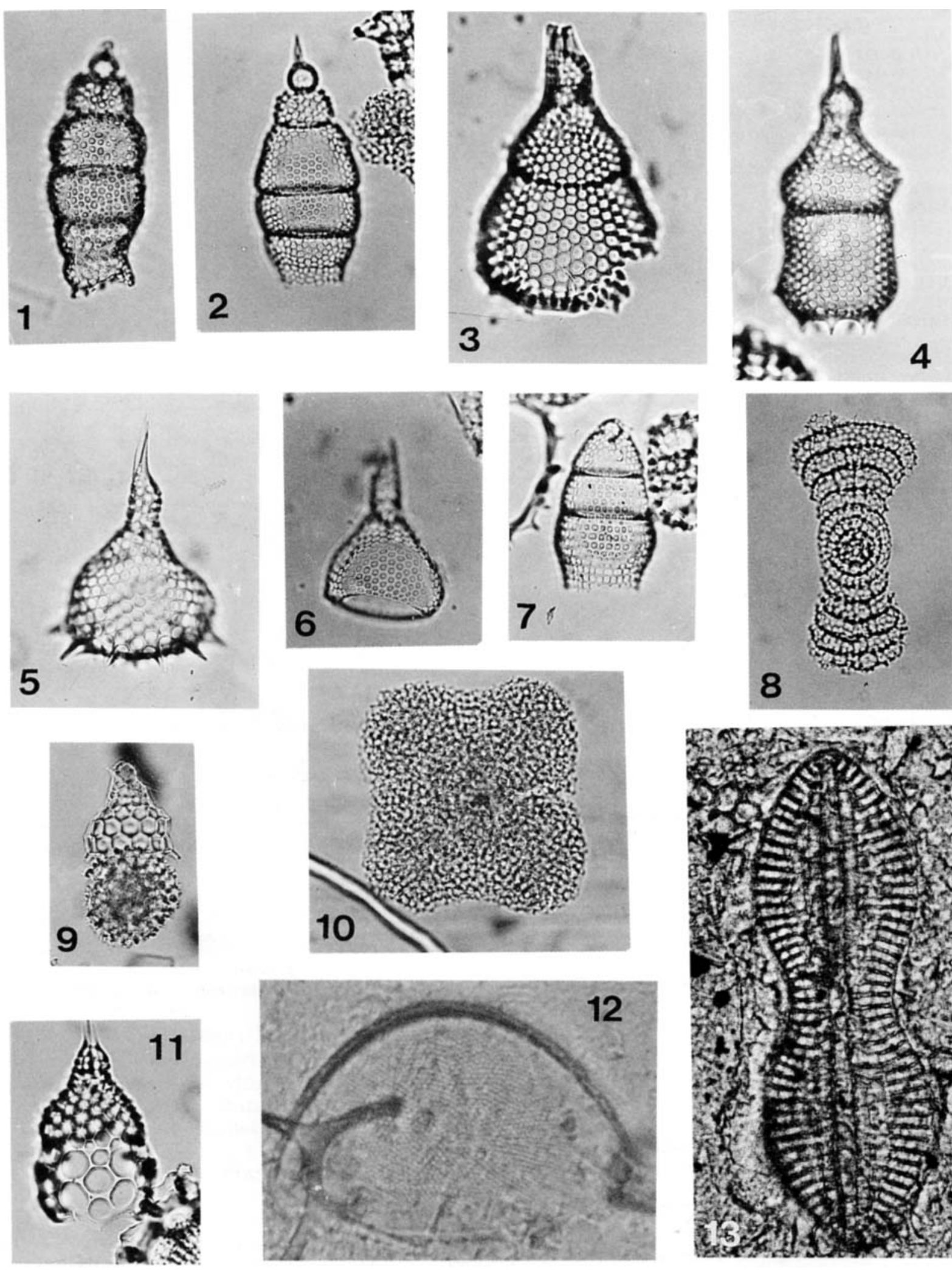

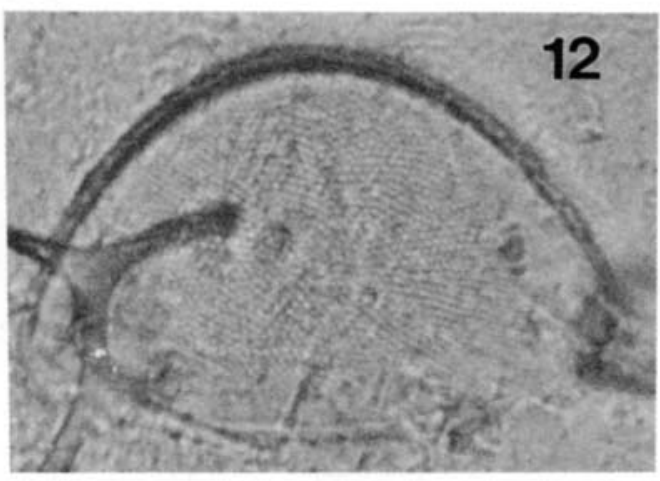

Plate 1
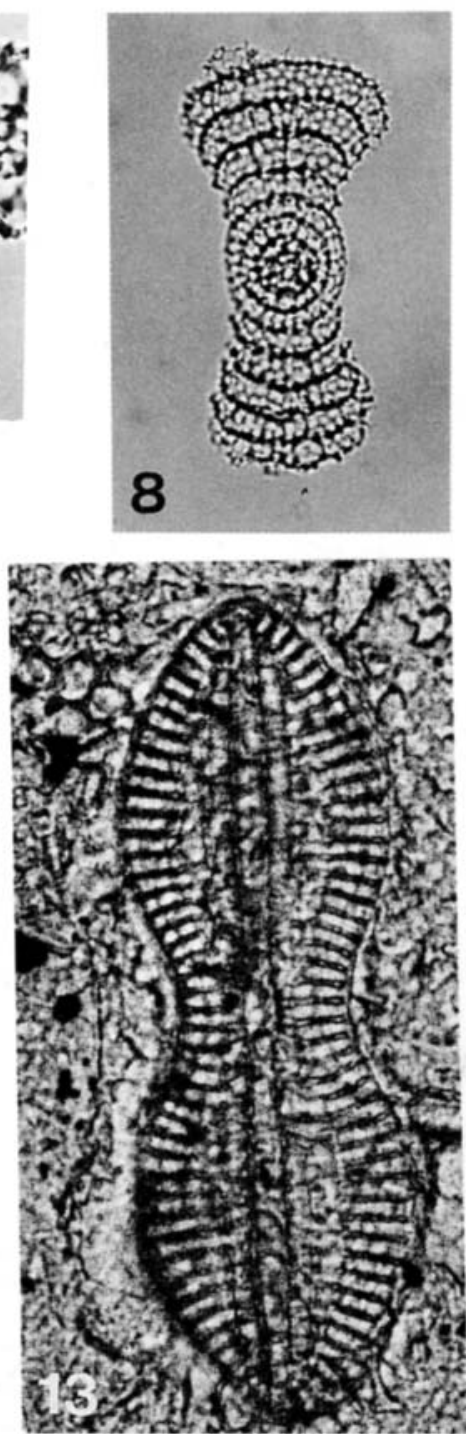


\begin{tabular}{|c|c|c|c|c|c|c|c|c|c|c|c|c|c|c|c|c|}
\hline Samples & 1 & 2 & 3 & 4 & 5 & 6 & 7 & 8 & 9 & 10 & 11 & 12 & 13 & 14 & 15 & $\mathrm{Z}$ \\
\hline $3 / 1 / 5-7$ & - & + & + & + & - & - & - & + & - & + & - & + & - & & & $\mathrm{I}$ \\
\hline $3 / 1 / 120-122$ & - & + & - & + & + & - & - & + & - & - & - & + & - & & & \\
\hline $3 / 1 / 145-147$ & - & + & - & + & + & - & - & + & + & - & - & - & - & & & \\
\hline $3 / 2 / 135-137$ & - & + & + & - & - & + & + & + & - & - & - & - & - & & & \\
\hline $3 / 2 / 140-142$ & - & t & + & + & - & + & - & + & - & - & + & - & - & & & \\
\hline $3 / 2 / 145-147$ & - & + & - & + & + & + & - & - & - & - & - & + & - & & & \\
\hline $3 / 3 / 5-7$ & - & + & + & + & + & + & - & + & - & - & - & + & - & & & \\
\hline $3 / 4 / 65-67$ & - & + & + & + & + & + & - & + & + & - & + & - & + & & & IIc \\
\hline $3 / 4 / 90-92$ & & + & + & + & + & + & - & + & + & + & + & - & + & & & \\
\hline $3 / 4 / 145-147$ & & - & + & + & + & + & + & + & - & - & + & + & - & & & \\
\hline $3 / 5 / 145-147$ & & + & + & + & + & - & - & + & + & - & - & + & - & & & \\
\hline $3 / 6 / 145-147$ & & + & - & - & + & - & - & + & + & - & - & - & - & & & \\
\hline $3 / 7 / 145-147$ & & - & - & - & - & - & - & - & - & - & - & - & - & & & \\
\hline $3 / 7 / 50-52$ & & + & - & + & + & - & - & + & + & - & - & - & - & & & \\
\hline $4 / 1 / 10-12$ & & - & - & - & + & - & - & + & + & - & - & + & - & - & & \\
\hline $4 / 1 / 50-52$ & & - & + & + & - & + & + & + & + & - & - & - & - & + & & $\mathrm{IIb}$ \\
\hline $4 / 1 / 100-102$ & & + & - & + & - & + & - & + & + & - & + & + & - & + & - & \\
\hline $4 / 1 / 120-122$ & & + & + & + & + & + & + & + & + & + & - & + & - & + & + & II \\
\hline $4 / 1 / 145-147$ & & + & + & - & + & - & - & + & + & - & - & + & - & + & + & \\
\hline $4 / 2 / 5-7$ & & - & + & + & + & + & + & + & - & - & - & + & - & + & + & \\
\hline $4 / 2 / 120-122$ & & + & + & + & + & - & - & + & + & - & + & + & + & + & + & \\
\hline $4 / 2 / 145-147$ & & - & + & + & + & - & - & + & + & - & - & + & - & + & - & III \\
\hline $4 / 3 / 145-147$ & & & - & - & - & - & - & - & + & - & - & - & - & - & - & \\
\hline $4 / 4 / 90-92$ & & & + & + & + & - & - & - & + & - & - & - & + & + & + & \\
\hline $4 / 4 / 120-122$ & & & - & + & + & - & - & - & + & - & - & - & - & + & + & \\
\hline $4 / 4 / 135-137$ & & & - & + & - & - & - & + & + & - & - & - & - & + & - & \\
\hline $4 / 4 / 145-147$ & & & + & - & - & - & - & - & - & - & - & - & - & + & - & \\
\hline $4 / 5 / 145-147$ & & & - & - & - & - & - & - & - & - & - & - & - & - & - & \\
\hline $4 / 6 / 145-147$ & & & + & + & - & - & - & + & + & - & - & + & - & + & + & \\
\hline $4 / 7 / 60-62$ & & & + & + & - & + & - & + & + & - & - & + & - & + & + & \\
\hline $4 / C C / 10-12$ & & & + & + & - & - & - & + & + & - & - & + & + & + & + & \\
\hline $5 / 1 / 5-7$ & & & - & + & - & - & - & + & + & + & - & + & - & + & + & \\
\hline $5 / 1 / 145-147$ & & & - & - & - & - & - & - & - & - & - & - & + & + & + & \\
\hline $5 / 2 / 145-147$ & & & + & - & - & - & - & + & + & + & - & + & + & + & + & \\
\hline $5 / 3 / 145-147$ & & & - & - & - & + & - & - & + & - & - & - & - & + & - & \\
\hline $5 / \mathrm{CC} / 5-7$ & & & + & + & - & + & - & + & + & - & - & + & - & - & + & \\
\hline $6 / 1 / 10-12$ & & & + & + & - & + & - & + & + & - & - & + & - & + & + & \\
\hline
\end{tabular}

$+=$ present; $-=$ searched for but not found. Species $1=$ Pseudoeunotia doliolus; $2=$ Rhizosolenia praebergonii; $3=$ Nitzschia reinholdii $; 4=N$. marina $; 5=N$. fossilis $; 6=$ Hemidiscus cuneiformis $; 7=$ Dipploneis $\mathrm{sp}$.; $8=$ Coscinodiscus nodulifer $; 9=C$. kolbei $; 10=C$. excentricus; $11=$ Biddulphia alternans; $12=$ Ethmodiscus sp.; $13=$ Rhizosolenia sp.; $14=$ Thalassiosira convexa $; 15=$ Nitzschia jouseae. Zone $\mathrm{I}=$ Nitzschia reinholdii zone; $\mathrm{II}=$ Rhizosolenia praebergonii zone with subzones a, b, c; III = Nitzschia jouseae zone.

Table 3. Diatom stratigraphy of Hole 709C.

davisiana, FAD of Theocorythium trachelium, and FAD of Lamprocyrtis neoheteroporos.

Stichocorys peregrina Interval Chronozone (non S. peregrina zone of Riedel \& Sanfilippo, 1970; emend. Riedel \& Sanfilippo, 1978; Johnson et al., 1989; emend. here).

Definition. LAD of Phormostichoartus doliolum to LAD of $S$. peregrina.
709C events included in this zone: LAD of Lychnodictyum audax.

Remarks. Johnson et al. (1989) define the base of this zone with the LAD of Phormostichoartus fistula Nigrini. However, in this study only one undoubted specimen of $P$. fistula was encountered. Therefore, the $P$. fistula Interval Chronozone of Johnson et al. (1989) is omitted here and the base of the $S$. peregrina Interval Chronozone is redefined here as the LAD of $P$. doliolum.

\section{Explanation of Plate 2}

Fig. 1. Rhizosolenia sp., sample 4/5/145-147, mag. $\times 1200$. Figs 2, 3. Rhizosolenia praebergonii Mukhina, sample $3 / 3 / 65-67$, mag. $\times 1200$. Fig. 4. Coscinodiscus nodulifer Schmidt, sample 3/1/120-122, mag. $\times 1300$. Fig. 5. Thalassiosira convexa Mukhina, sample 4/CC/10-12, mag. $\times 1180$. Fig. 6. Nitzschia jouseae Burkle, sample 4/2/145-147, mag. $\times 1300$. Fig. 7. Nitzchia fossilis (Frenguelli), sample 3/1/145-147, mag. $\times 1100$. Fig. 8. Nitzchia reinholdii Kanaya \& Koizumi, sample 4/1/50-52, mag. $\times 1200$. Fig. 9. Nitzschia marina Grunow, sample 3/1/5-7, mag. $\times 650$. 

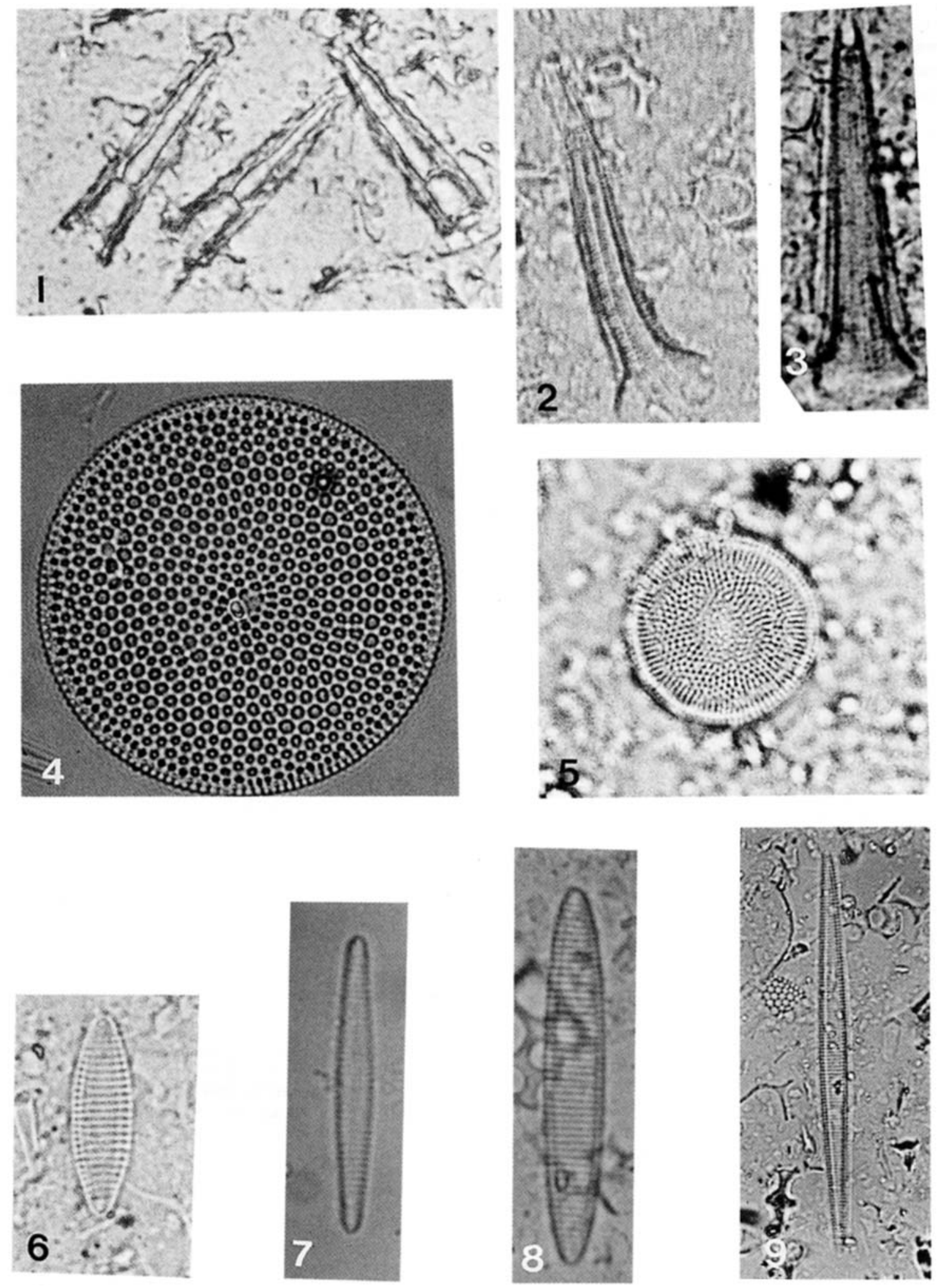

Plate 2 
Phormostichoartus doliolum Interval Chronozone (Johnson et al., 1989).

Definition. LAD of Anthocyrtidium prolatum to LAD of P. doliolum.

709C events included in this zone: FAD of Amphirhopalum ypsilon.

The variations between the zonal scheme proposed by Johnson et al. (1989) and the scheme derived from the present study, can be attributed to oceanographical differences of the respective locations. Whereas Hole 709C is located $4^{\circ} \mathrm{S}$ in an area subject to equatorial upwelling, the three cores examined by Johnson et al. (1989) containing equivalent aged sediments, were all taken south of the hydrochemical front (Wyrtki, 1973). As already mentioned, this front separates high and low productivity areas respectively to the north and south of $10^{\circ} \mathrm{S}$.

The diatom results from the present study support and refine those found by Mikkelsen (1990) with only one exception. Mikkelsen's study of 709C located the FAD of Pseudoeunotia doliolus at 3/3/92-93-3/4/92-93. In the present study, however, this species was not encountered. The proximity of the FAD of $P$. doliolus in Mikkelsen's study to the top of the section under examination here is very close. Other species found at this point are poorly preserved and perhaps this accounts for the absence of $P$. doliolus. A further possible explanation may be attributable to the similarity of $P$. doliolus and Nitzschia fossilis (Frenguelli). With the poor preservational state and the similarity of these two species, it is plausible that an identification error may have been made.

In addition to biostratigraphically useful fossils, the presence of other diatom species were also noted. Nitzschia marina Grunow, Hemidiscus cuneiformis Wallich, Coscinodiscus nodulifer Schmidt, C. kolbei Jouse, $C$. excentricus Ehrenberg, Ethmodiscus sp., and Rhizosolenia sp. occurred throughout the studied section, but Dipploneis sp. and Biddulphia alternans Van Heurck exhibit restricted ranges.

\section{CONCLUSION}

From Johnson (1990), Mikkelsen (1990) and the present study, it appears that Site 709 is unsuitable as a high resolution biostratigraphical reference section for siliceous microfossils. This is owing to a number of reasons. The poor preservation at certain horizons limits the precision with which biodatums can be identified. The scarcity and/or absence of some important stratigraphical marker species such as Pterocanium prismatium, Pterocorys zancleus, Theocorythium vetulum, Anthocyrtidium ehrenbergi (as noted by Johnson, 1990), A. pliocenica (Seguenza), Phormostichoartus fistula and Pseudoeunotia doliolus (present study), is limiting. (Spongaster tetras which was recorded as very rare or absent in 709A (Johnson, 1990), was found to be more abundant in 709C).

Nevertheless, Site 709 is still very useful in terms of calcareous biostratigraphy and palaeoceanographical studies of both calcareous and siliceous microfossils. This is particularly so as oxygen isotope callibrated dates are being

\begin{tabular}{llcc}
\hline \multicolumn{1}{c}{ Species } & \multicolumn{1}{c}{ Sample } & Depth* & Age(Ma) \\
\hline FAD Anthocyridium angulare & $3 / 1 / 5-7$ & 15.45 & 1.65 \\
LAD Theocorythium vetulum & $3 / 2 / 145-147$ & 18.35 & 2.00 \\
LAD Anthocyrtidium jenghisi & $3 / 4 / 75-77$ & 20.65 & 2.27 \\
FAD Theocalyptra davisiana & $3 / 4 / 145-147$ & 21.35 & 2.33 \\
FAD Theocorythium trachelium & $3 / 4 / 145-147$ & 21.35 & 2.33 \\
FAD Lamprocyris neoheteroporos & $3 / 5 / 75-77$ & 22.15 & 2.40 \\
\hline
\end{tabular}

*depth (mbsf) as recorded by the Shipboard Scientific Party (1988).

Table 4. Ages for some radiolarian datum levels identified in Hole 709C. Based on Shackleton \& Hall (1990) oxygen isotope data.

developed for Hole $709 \mathrm{C}$ from data presented by Shackleton \& Hall (1990). Dates derived from oxygen isotope and calcareous microfossil data, downhole to sample $3 / 7 / 10-12$, are assigned in Table 4.

\section{ACKNOWLEDGEMENTS}

This research was funded by an NERC Special Topic grant, Kevin Kennington is funded by an NERC Research Studentship.

\section{Manuscript received March 1994 \\ Manuscript accepted February 1995}

\section{REFERENCES}

Alexandrovich, J. M. 1989. Radiolarian biostratigraphy of ODP Leg 111, Site 677, eastern equatorial Pacific. Proceedings of the Ocean Drilling Program, Scientific Results, 111: 245-262.

Andrews, J. E., Funnell, B. M., Jickells, T. D., Shackleton, N. J., Swallow, J. E., Williams, A. C. \& Young, K. A. 1990. Preliminary assessment of cyclic variations in foraminifers, barite, and cadmium/calcium ratios in early Pleistocene sediments from Hole $709 \mathrm{C}$ (equatorial Indian Ocean). Proceedings of the Ocean Drilling Program, Scientific Results, 115: 611-619.

Barron, J. A. 1985. Miocene to Holocene planktic diatums. In Bolli, H. M., Saunders, J. B. \& Perch-Nielsen, K. (Eds), Plankton Stratigraphy, 763-809. Cambridge University Press, Cambridge.

Boersma, A. 1990. Late Oligocene to Late Pliocene benthic Foraminifers from depth traverses in the central Indian Ocean. Proceedings of the Ocean Drilling Program, Scientific Results, 115: $315-379$.

Burckle, L. H. 1972. Late Cenozoic planktonic diatom zones from the eastern equatorial Pacific. Nova Hedwigia, 39: 217-246.

Caulet, J. P. 1979. Les depots à Radiolaires d'âge pliocene supérieur à pleistocene dans l'océan Indien central: nouvelle zonation biostratigraphique. Memoiredu Musée National de la Histoire Naturelle de Paris, Série C, 43: 119-141.

Caulet, J. P. \& Nigrini, C. 1988. The genus Pterocorys (Radiolaria) from the tropical late Neogene of the Indian and Pacific Oceans. Micropaleontology, 34: 217-235.

Fenner, J. \& Mikkelsen, N. 1990. Eocene-Oligocene diatoms in the western Indian Ocean: taxonomy, stratigraphy and paleoecology. Proceedings of the Ocean Drilling Program, Scientific Results, 115: 433-463.

Fornaciari, E., Raffi, I., Rio, D., Villa, G., Backman, J. \& Olafsson, G. 1990. Quantitative distribution patterns of Oligocene and Miocene calcareous nannofossils from the western equatorial Indian Ocean. Proceedings of the Ocean Drilling Program, Scientific Results, 115: 237-254.

Goll, R. M. 1980. Pliocene-Pleistocene radiolaria from the East Pacific Rise and the Galapogos spreading centre; DSDP Leg 54. Initial Reports of the Deep Sea Drilling Project, 54: 425-454.

Haslett, S. K. 1992. Plio-Pleistocene radiolarian biostratigraphy of the tropical Indian Ocean (ODP Hole 709C). In Haslett, S. K. 
(Ed.), Siliceous Microfossils (abstracts), 8. School of Environmental Sciences, University of East Anglia, Norwich.

Haslett, S. K., Funnell, B. M., Bloxham, K. S. \& Dunn, C. L. 1994. Plio-Pleistocene palaeoceanography of the tropical Indian Ocean (ODP Hole $709 \mathrm{C}$ ): radiolarian and $\mathrm{CaCO}_{3}$ evidence. Journal of Quaternary Science, 9: 199-208.

Haslett, S. K. \& Kersley, C. L. 1995. Early Pleistocene planktonic foraminifera from the tropical Indian Ocean. Microscopy \& Analysis, 46 (March 1995): 25-28.

Haslett, S. K. \& Robinson, P. D. 1991. Detecting radiolaria in the field. Journal of Micropalaeontology, 10: 22.

Johnson, D. A. 1990. Radiolarian biostratigraphy in the central Indian Ocean, Leg 115. Proceedings of the Ocean Drilling Program, Scientific Results, 115: 395-409.

Johnson, D. A. \& Knoll, A. H. 1975. Absolute ages of Quaternary radiolarian datum levels in the equatorial Pacific. Quaternary Research, 5: 99-110.

Johnson, D. A., Schneider, D. A., Nigrini, C. A., Caulet, J. P. \& Kent, D. V. 1989. Pliocene-Pleistocene Radiolarian events and magnetostratigraphic calibrations for the tropical Indian Ocean. Marine Micropaleontology, 14: 33-66.

Kazarina, G. K. 1975. Diatom zones in the sediments of the eastern tropical region of the Indian Ocean. Okeanol. Akad. Nauk, 15: 1073-1078.

Kennington, K. 1992. Plio-Pleistocene diatom biostratigraphy of ODP Hole $709 \mathrm{C}$ (equatorial Indian Ocean). In Haslett, S. K. (Ed.), Siliceous Microfossils (abstracts), 11. School of Environmental Sciences, University of East Anglia, Norwich.

Leinen, M., Cwienk, D., Heath, D., Biscaye, G. R., Kolla, P. E., Thiede, J. \& Dauphin, J. P. 1986. Distribution of biogenic silica and quartz in recent deep-sea sediments. Geology, 14: 199-203.

Matsuoka, H. \& Okada, H. 1990. Time-progressive morphometric changes of the genus Gephyrocapsa in the Quaternary sequence of the tropical Indian Ocean, Site 709. Proceedings of the Ocean Drilling Program, Scientific Results, 115: 255-270.

Mikkelsen, N. 1990. Cenozoic diatom biostratigraphy and paleoceanography of the western equatorial Indian Ocean. Proceedings of the Ocean Drilling Program, Scientific Results, 115: 411-432.

Mullineaux, L. S. \& Westberg-Smith, M. J. 1986. Radiolarians as paleoceanographic indicators in the Miocene Monterey Formation, Upper Newport Bay, California. Micropaleontology, 32: $48-71$.

Nigrini, C. 1971. Radiolarian zones in the Quaternary of the equatorial Pacific Ocean. In Funnell, B. M. \& Riedel, W. R. (Eds), The Micropalaeontology of Oceans, 443-461. Cambridge University Press, Cambridge.
Nigrini, C. 1985. Radiolarian biostratigraphy in the central equatorial Pacific, Deep Sea Drilling Project Leg 85. Initial Reports of the Deep Sea Drilling Project, 85: 511-551.

Nigrini, C. \& Caulet, J. P. 1988. The genus Anthocyrtidium (Radiolaria) from the tropical late Neogene of the Indian and Pacific Oceans. Micropaleontology, 34: 341-360.

Okada, H. 1990. Quaternary and Paleogene calcareous nannofossils, Leg 115. Proceedings of the Ocean Drilling Program, Scientific Results, 115: 129-174.

Premoli-Silva, I. \& Spezzaferri, S. 1990. Paleogene planktonic Foraminifera biostratigraphy and paleoenvironmental remarks on Paleogene sediments from Indian Ocean sites, Leg 115. Proceedings of the Ocean Drilling Program, Scientific Results, 115: $277-314$.

Riedel, W. R. \& Sanfilippo, A. 1970. Radiolaria, Leg 4, Deep Sea Drilling Project. Initial Reports of the Deep Sea Drilling Project, 4: $503-575$.

Riedel, W. R. \& Sanfilippo, A. 1978. Stratigraphy and evolution of tropical Cenozoic radiolarians. Micropaleontology, 24: 61-96.

Rio, D., Fornaciari, E. \& Raffi, I. 1990. Late Oligocene through early Pleistocene calcareous nannofossils from western equatorial Indian Ocean (Leg 115). Proceedings of the Ocean Drilling Program, Scientific Results, 115: 175-235.

Sanfilippo, A., Westberg-Smith, M. J. \& Riedel, W. R. 1985. Cenozoic radiolaria. In Bolli, H. M., Saunders, J. B. \& Perch-Nielsen, K. (Eds), Plankton Stratigraphy, 631-712. Cambridge University Press, Cambridge.

Schrader, H. J. 1974. Cenozoic marine planktonic diatom stratigraphy of the tropical Indian Ocean. Initial Reports of the Deep Sea Drilling Project, 24: 887-967.

Shackleton, N. J. \& Hall, M. A. 1990. Pliocene oxygen isotope stratigraphy of Hole $709 \mathrm{C}$. Proceedings of the Ocean Drilling Program, Scientific Results, 115: 529-538.

Shipboard Scientific Party. 1988. Site 709. Proceedings of the Ocean Drilling Program, Initial Reports, 115: 459-588.

Spaulding, S. A., Bloemendal, J., Hayashida, A., Hermelin, J. O. R., Kameo, K., Kroon, D., Nigrini, C. A., Sato, T., Steens, T. N. F., Takayama, T. \& Troelstra, S. R. 1991. Magnetostratigraphic and biostratigraphic synthesis, Leg 117, Arabian Sea. Proceedings of the Ocean Drilling Program, Scientific Results, 117: 127-145.

Theyer, F., Mato, C. Y. \& Hammond, S. R. 1978. Paleomagnetic and geochronologic calibration of latest Oligocene to Pliocene radiolarian events, equatorial Pacific. Marine Micropaleontology, 3: 377-375.

Wyrtki, K. 1973. Physical oceanography of the Indian Ocean. In Zeitzschel, B. \& Gerlach, S. A. (Eds), The Biology of the Indian Ocean, 18-36. Springer-Verlag, New York. 